\title{
Seamless Access to Sensor Networks for Enhanced Manufacturing Processes
}

\author{
Kostas Kalaboukas ${ }^{1}$, Borislav Jerabek ${ }^{2}$, Rok Lah $^{2}$, and Freek van Polen ${ }^{3}$ \\ ${ }^{1}$ SingularLogic, N. Ionia, Attica, Greece \\ kkalaboukas@singularlogic.eu \\ ${ }^{2}$ Gorenje, Partizanska cesta 12, 3320, Velenje, Slovenia \\ \{boro.jerabek, rok.lah\} @gorenje.si \\ ${ }^{3}$ Sense B.V., Lloydstraat 5, Rotterdam, Netherlands \\ freekesense-os.nl
}

\begin{abstract}
WSNs are largely deployed nowadays in "smart factories". However, their smooth operation is hindered by device heterogeneity and lack of integration with the current IT systems. The SIMPLE project aims at seamless inter-working of sensors, actuators, tags and devices with business IT systems and distributed control systems towards simpler integrated manufacturing processes. SIMPLE delivers a novel hierarchical network architecture, based on the envisioned manufacturing processes and reflecting the functional hierarchy of aggregations of goods or production processes found in the manufacturing use case.
\end{abstract}

Keywords: integrated manufacturing processes, network design, sensor networks, smart factories.

\section{Introduction}

Wireless Sensor Networks (WSNs) have been identified as one of the most important technologies for the $21^{\text {st }}$ century. The deployment of WSN in "smart factories" applications is already a fact and has attracted the interest of the research community and the electronics development giants worldwide. In a medium sized manufacturing plant over an area of $10000 \mathrm{~m}^{2}, 5000$ pressure sensors, 1000 temperature sensors and 500 sensors of other types exist on average [1]. However, significant problems hinder their wide exploitation: this great number of sensors includes sensors of different type (e.g. temperature, pressure, RFID reader), operating on different device platforms with different capabilities and communication characteristics. This heterogeneity prevents the simple, uniform access of information throughout the sensor cloud and its seamless integration with the business IT systems, which will be more and more needed, as the number of cheap multi-purpose sensors proliferates and even more reconfigurable sensor nodes are under development [2].

The seamless inter-working of sensors, actuators, tags and devices with business IT systems (e.g., ERP, WMS, MES) and distributed control systems towards "simpler" integrated manufacturing processes is at the core of the SIMPLE ${ }^{1}$ project [3].

\footnotetext{
${ }^{1}$ Self-organizing Intelligent Middleware Platform for manufacturing and Logistics Enterprises.

C. Emmanouilidis, M. Taisch, D. Kiritsis (Eds.): APMS 2012, Part II, IFIP AICT 398, pp. 25-32, 2013.

(C) IFIP International Federation for Information Processing 2013
} 
SIMPLE aims at designing and developing a flexible sensor network architecture and a middleware solution in order to compensate for the current lack of open-source and commercial solutions capable of monitoring the state of shipments at different grouping levels (e.g. at the crate and case levels) and, more generally, of tracing goods along the whole supply chain. The vision is to enable information collection from all over the manufacturing plant in a uniform way without having to consider the heterogeneity of the underlying infrastructure.

The present paper starts from the analysis of the current manufacturing processes (in section 2), which was carried out to create a concrete list of requirements, and reports the improvements, that an integrated solution can bring in section 3.To achieve these objectives and meet the identified requirements, a hierarchical network architecture has been designed and is presented in section 4. It reflects the functional hierarchy of aggregations of goods or production processes found in the manufacturing use case. Conclusions are drawn in section 5.

\section{Manufacturing “As-is" Situation}

To capture the functionality that can significantly simplify the manufacturing process, first the currently adopted white goods manufacturing process was analysed, based on input provided by Gorenje ${ }^{2}$. Currently, white goods manufacturing consists of 4 main phases:

Phase A: Framework Construction. All mechanic parts of the product are being assembled. The parts are picked from the warehouse and transferred to the production centre. There, a fully automated assembly line is responsible for putting together all parts to create the framework of the product.

Phase B: Electronic parts assembly. The framework goes to the second phase, where all electronic parts, circuits, accessories, control mechanisms, etc. are manually inserted. Similar to the mechanic parts, the electronics, are picked by the warehouse, exit it and transferred to the production center. Once assembled, the final product enters the testing phase.

Phase C: Testing. According to the results and the severity of the failures (if found), the product may have one of the following paths:

- $\quad$ The product has no failures and ready to be released. The product goes to the warehouse of final products for further delivery.

- $\quad$ The product has minor failures. In this case, the technical team disassemblies the product. All parts then are returned to the materials warehouse.

- $\quad$ The product has severe failures. The product is being disassembled and the technical team checks all parts separately. Those that are reusable go to the materials warehouse and the damaged ones are destroyed.

Phase D: After Sales Support. When the product has been sold, automated management support can still be provided. Within regular intervals, the appliances perform self-testing and upon detecting a malfunction, an alert message is sent or an error code

${ }^{2}$ Gorenje is one of the partners of the SIMPLE project consortium and the largest Slovenian manufacturer of white goods (larger home appliances). 
appears on the built-in led display. The after-sales support office is informed either by the client or automatically, so that the problem gets fixed.

\section{Manufacturing "To-be" Situation}

Through SIMPLE the manufacturing process is being automated in various phases allowing for easy monitoring of incoming/outgoing goods in the warehouse, as well as better tracking of the production and product-in-use phases. The modifications that were considered necessary for each manufacturing phase are described next:

Phase A: Framework Construction. A visual camera could test the position of the part to be assembled, the part type, as well as any possible anomalies.

Phase B: Electronic Parts assembly. Parts will be RFID-tagged in the warehouse and RFIDs will be registered in the SIMPLE server. A motion sensor will register parts transportation to the production center. After assembly, the final product is being tagged and the aggregated RFID is registered in the SIMPLE server.

Phase C: Testing. Depending on the testing result, the following SIMPLE intersection points can be triggered:

- The product has no failures. The product will be released, while a motion sensor will register the event of the product being ready for delivery.

- The product has minor failures. All parts will contain this information and enter the warehouse. A motion sensor will also register this event.

- $\quad$ The product has severe failures. The product is disassembled and the aggregated tag is being destroyed. The reusable parts are led to the warehouse and this event is recorded through a motion sensor. Each (tagged) part also contains the severe failures history and "reusable" status. The tags of the non-reusable parts are also deleted from SIMPLE. Additionally, integration with the ERP system can lead to automatic creation of material consumption reports and warehouse documents (when a part enters or leaves it, as well as automatically tracking the production process).

Phase D: After Sales Support. The product has an embedded Automated Testing Tool (ATE), which is beyond the scope of SIMPLE.

According to the envisioned manufacturing scenario, a number of system requirements have been derived. The requirements, related to the network architectural design, which is at the focus of this paper, are the following:

- $\quad$ Collection, filtering and processing of the readings from a variety of sensors, tags and sensor networks (e.g., RFID, temperature, pressure, humidity), and accordingly delivering them as reports.

- Mapping and correlation of the above reports to business events associated with user-defined business contexts and processes for integrated manufacturing. RFID tags will allow for accurate and automated association of manufacturing processes and items with wider business processes.

- Enabling configuration and invocation of actuating services in machines, devices and actuators.

- Provision of techniques and graphical tools for managing sensors, devices, as well as business contexts and processes. 


\section{The SIMPLE Solution}

SIMPLE is understood as a middleware platform and as such, it shall be a software layer operating on every device that is part of a SIMPLE application, and form the bridge among these devices capitalizing on emerging works on programming sensor nodes [4]. To efficiently face the diversity of devices mainly with respect to their hardware (processing and storage) capabilities, we have reviewed the devices usually involved in a manufacturing plant and we have organized them in classes, prior to designing the overall SIMPLE network and node architecture. Then, to address the scalability issues arising in such systems, we have designed a hierarchical network architecture that discriminates different hierarchy layers and specifies the functionality (including the middleware, networking and application functionality) that the device belonging to each layer should execute. It is worth stressing that there is no oneto-one relation between device class and layers, since a device belong to a specific class depending on its hardware capabilities while it plays the role of a layer $-\mathrm{x}$ device depending on the functionality it supports. Finally, to guide the development of the SIMPLE system, based on the network architecture and the specified functionality per layer, the SIMPLE node architecture is drawn.

\subsection{SIMPLE Nodes Classification}

The SIMPLE devices are classified according to hardware limitations as follows:

Class 0 Devices: RFID Tags. Radio-frequency identification (RFID) makes use of radio signals to exchange data between a reader and an electronic tag attached to an object, for the purpose of identification and tracking. RFID tags can be read by the RFID reader, in case of short distance, antenna matching and possible signal propagation. The RFID tags contain an integrated circuit for storing and processing information, modulating and demodulating a radio signal and an antenna for receiving and transmitting the signal. RFID tags can either just have a fixed ID or they could in addition also have a read-write memory from and to which the reader is able to read and write information.

In case of RFID tags the RFID readers that communicate to the individual tags are the interfaces to the rest of the SIMPLE system. The RFID readers collect the information from the tags, store it locally and, if required, communicate the information to the rest of the SIMPLE system and write new information to the RFID tags via the middleware interface. The main functions that these tags types support are tag inventorying and optimizing, reading/writing, locking and killing.

Class 1 Devices: WSN Nodes. WSNs are distributed networked systems composed of embedded computing nodes, each one being equipped with a processing unit (typically a microcontroller), a wireless communication interface, sensor(s) board and a battery. The wireless sensor networks typically make use of ad-hoc multi-hop networking schemes, which means that all nodes operate as routers, as well as they should be able to join and leave the network without any disruptions to the network operation. 
Class 2 Devices: Hand-held RFID Readers. While RFID readers by themselves may be very small and attached to WSN nodes, hand-held RFID readers are included in a separate class, mainly due to their specific role in the SIMPLE systems. This class will typically be able to engage in user interaction, while also being able to read RFID devices, and thus be a powerful tool in a SIMPLE system for configuring installations. This type of device shall typically be used to add RFID tags to the system, to aggregate groups of devices, and to attach RFID tags to objects that need to be represented in the system.

Class 3 Devices: Hand-held and Embedded Devices. Hand-held and embedded devices, even personal and general-purpose devices, such as smartphones, are expected to be present in manufacturing environments. Devices of this type are marked by a high degree of connectivity. In SIMPLE, this class of devices could fulfill several different functions. First, their ubiquitous presence and high connectivity gives them the potential to be powerful ad-hoc routing hops. Second, they could operate as mobile sinks for any WSNs in the vicinity, as well as gather their own sensor data, with any sensors they might have equipped themselves. Third, these devices typically are designed for user interaction, and so could be used by any SIMPLE application.

Class 4 Devices: Desktops and Laptops. Desktops and laptops feature great processing power and memory, which allow them to sit in the background behind a sink node and operate on data that have been collected by a WSN. Within SIMPLE, desktops and laptops will be a readily available source of computing power and user interaction. Therefore, they could store and process large amounts of data in a local fashion. Additionally, they will be the most obvious choice for SIMPLE applications to interface with users, allowing them to monitor processes and configure a SIMPLE installation.

Class 5 Devices: Servers and "The Cloud". With recent developments in cloud computing and the increase in cloud computing services being offered, a virtually unlimited amount of computing power and storage room becomes available. Recently, however, services have become available that focus specifically on the storage and processing of sensor data. Within SIMPLE, this class of devices will be able to store and backup all data gathered in an installation. While the capabilities of this class of devices are virtually unlimited, they do have their limitations in that the amount of communication required may introduce lag in the system. Therefore, a the kind of data being stored and processed locally (typically recent data that may become relevant very quickly), as well as the kind of data being transported to the cloud for storage and processing should be carefully chosen.

\subsection{The SIMPLE Network Architecture for the Manufacturing Use Case}

The SIMPLE hierarchical network architecture can be applied to a variety of cases and is specifically useful for large and heterogeneous WSN systems. However, as the focus of this paper is on the enhancement of the manufacturing process, we concentrate on its operation for the manufacturing ("to-be") use case. The SIMPLE layered network architecture is presented in Fig. 1. 


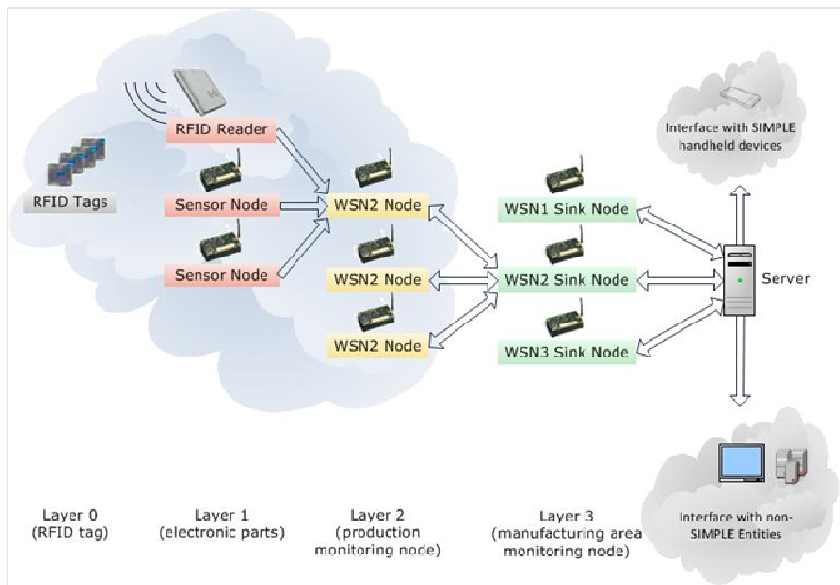

Fig. 1. The SIMPLE high level network architecture for the manufacturing case

In Phase A, the RFID reader and the camera or other visual inspection devices are considered as layer-1 devices. A layer-2 node (acting as information aggregator) receives the sensed data and can create a list of the mechanical parts that will be used for a specific good and their condition. This list is created either periodically or upon user request.

In Phase B, the RFID reader and the camera are regarded as layer- 1 nodes.

In Phase $\mathrm{C}$, testing, once a new device has been produced, a layer- 2 node or another layer -1 node inspects the product. The result is announced to the layer- 2 node, which has also collected information about the parts that the product consists of. This node now creates a product ID, associates it with the RFIDs of the parts it consists of and creates a message towards the server to inform that a new product is ready providing the details of its sub-parts. In the case of failure, the product is disassembled and the layer-2 node generates a different message providing the list of RFIDs of the parts. If any of them is no longer reusable, its RFID is sent to the server marked as "to be destroyed".

In Phase D, after sales support, it is the embedded Automated Testing Tool (ATE) that assists in problem fixing and SIMPLE is not involved.

From a communication perspective, layer-3 nodes are assumed to be installed in large manufacturing areas and are responsible for collecting information from sensor nodes regarding one or multiple production lines, as well as area-related information (e.g. temperature, humidity, light). Layer-3 nodes are also acting as gateways to the enterprise server.

Layer 1, 2 and 3 nodes are either in one or multi-hop distance between them, thus the need for realizing a WSN routing protocol arises. As regards the location, all nodes will most probably be placed in fixed locations, thus the exact coordinates can be statically configured.

As regards functionality,

1. layer-1 nodes sense data (RFID reader, anomaly detection, visual inspection),

2. layer-2 nodes generate product IDs and associate them with the IDs of the parts that form the product,

3. layer-3 nodes aggregate information regarding one or more production lines and areas and connects the layer 2 nodes to the server. 


\subsection{The SIMPLE-Node Architecture}

The high level architecture of the SIMPLE node following an OSI approach is shown in Fig. 2. The SIMPLE research efforts are focused on the layers above the MAC layer.

At the network layer, a location manager module will exist which will either become aware of the nodes location through GPS or will execute a localization technique.

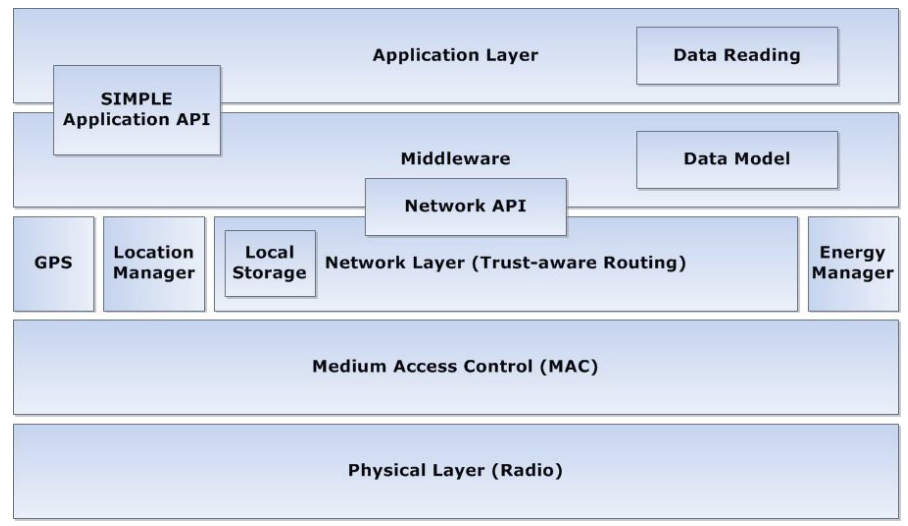

Fig. 2. The SIMPLE OSI-aligned node architecture

At the same OSI layer, the trust-aware routing block will undertake the responsibility to define the path of any message from any SIMPLE node towards the SIMPLE sink node. In addition, a trust management system will guarantee the detection and avoidance of malicious nodes. The trust information will be kept in the Local Storage block per one-hop neighbor and will be combined with the location information to define the next-hop node.

The Energy manager monitors the remaining energy of the node for two purposes: first, to inform the upper layers in case the remaining energy is running low and second to inform any layer (e.g. routing) about the current energy level so that it takes this information into account to reduce the overall energy consumption.

The middleware layer contains the data model, containing all necessary data for the manufacturing case. The data fields specified in this model are a listing of the data type stored in the middleware layer. Consequently, all functionality offered to the SIMPLE applications shall ultimately operate on these data fields. The data model provides functionality to store sensor data, as well as device IDs and aggregations between devices. Also, the data model is able to represent real world objects, which are not SIMPLE devices, and associations between SIMPLE devices and objects. For the manipulation of these basic data fields, low-level data manipulation functions are defined, which are part of this data model. 
The SIMPLE Application API forms the bridge between the SIMPLE middleware layer and SIMPLE applications. The API allows applications to pose queries and subscribe to specific events. Moreover, the API allows the application to set certain conditions on the validity of the data that are to be returned. These conditions can be on the value of data itself, but also on the geographic region from which the data originate, the time at which the data were gathered, or the confidence the middleware layer has that indeed it is valid data.

\section{Conclusions}

SIMPLE has been presented as an integrated solution to the manufacturing automation. SIMPLE aims at delivering answers to as yet unsolved problems and products long needed for the commercialization of sensor networks in large-scale manufacturing and logistics applications. These objectives are met by progressing the state-of the art in three key research areas, namely in a) energy efficient and secure sensor routing, b) middleware technologies and c) sensor's network architecture design.

The present work was focused on the third field, elaborating the manufacturing process, continuing with the heterogeneous hardware classification, the SIMPLE high-level node architecture, supporting the aforementioned situation and ending in the SIMPLE network architecture design presentation. Obviously, the SIMPLE architecture definitely supports fully automated manufacturing operation.

The designed architecture, along with the defined functionality, will be mapped to a real-life test-bed, demonstrating the complete functionality of the SIMPLE system. The proposed developments will be able to provide traceability of the components and the products, store the history of each product and provide monitoring and transparent trace data to the manufacture plants, the warehouse, the distribution stores and the final customers. The test-bed will be set up in the Gorenje premises.

Acknowledgement. The work presented in this paper was partially funded by the ARTEMIS SIMPLE project.

\section{References}

1. Mols, D.: Making Measurement Count. Control Engineering Europe Journal, 24-26 (June/July 2009)

2. http://www.artemis-smart.eu/home.aspx

3. http://www.simple-artemis.eu/

4. Mottola, L., Picco, G.: Programming Wireless Sensor Networks: Fundamental Concepts and State of the Art. ACM Computing Surveys (2011) 